\title{
PSICOLOGIA E RESILIÊNCIA: Uma revisão de literatura
}

\author{
Psychology and resilience: A literature review
}

\author{
Rosana Angst \\ Acadêmica do Curso de Psicologia (PUCPR), Curitiba, PR - Brasil, e-mail: roangst@gmail.com
}

\begin{abstract}
Resumo
Diante da situação mundial atual que se caracteriza pela alta ocorrência de eventos aversivos no decorrer do desenvolvimento, algumas pessoas passam por situações traumáticas e a superam saindo fortalecidas, e outras não conseguem recuperar-se da situação ocorrida. Um dos motivos para que cada indivíduo lide de formas diferentes com problemas semelhantes está relacionado com o conceito de resiliência. A resiliência caracteriza-se pela capacidade de um determinado sujeito ou grupo passar por uma situação adversa, conseguir superá-la e sair dela fortalecido. Pessoas resilientes apresentam características como autoestima positiva, habilidades de dar e receber em relações humanas, disciplina, responsabilidade, receptividade e tolerância ao sofrimento. Ao passar por uma situação traumática, muitas pessoas tendem a procurar ajuda profissional para orientá-las nesse contexto adverso, sendo o psicólogo um desses profissionais que possuem formação adequada para fornecer a ajuda necessária. Os estudos sobre a resiliência são de grande contribuição para o trabalho do psicólogo, pois ao conhecer a resiliência, será possível ver um indivíduo como capaz de procurar recursos para superar as adversidades, não sendo somente um observador passivo de sua história, e sim como alguém capaz de buscar recursos em si mesmo e no ambiente que o rodeia para a resolução de conflitos.
\end{abstract}

Palavras-chave: Resiliência. Resiliência e psicologia. Fatores de proteção. Fatores de risco.

\begin{abstract}
Before the actual world situation characterized by the high occurrence of aversive events during the development, some people go through traumatic situations and get over them stronger, and others can't recover from the occurred situation. One of the reasons that explain why each person deal differently before similar situations is related to the concept of resilience. Resilience is characterized by a person or group's ability to go through an adverse situation, get over it, and become stronger after the event. Resilient people present characteristics as high self-esteem,
\end{abstract}


ability to give and receive in human relationships, discipline, responsibility, receptivity and tolerance. When people go through a traumatic situation, they tend to seek professional help to guide them, and a psychologist is a professional able to provide it. Studies about resilience are in general a great way to help psychologist's work, because when they are familiar with the concept, they won't perceive their patient as a passive observer of his history, but someone able to search resources in himself and on the environment around him to solve a problem.

Keywords: Resilience. Resilience and psychology. Risk factors. Protection factors.

\section{INTRODUÇÃO}

No contexto mundial em que vivemos hoje, é impossível não passarmos por qualquer tipo de adversidade durante a vida. Desde uma briga conjugal e término de um relacionamento, a morte de um ente querido, até a perda de casas em um terremoto ou enchente. Porém, determinados eventos podem ser traumáticos para uma pessoa a ponto de impossibilitála a seguir sua vida, e para outra esse processo é difícil, mas é elaborado, o possibilitando a continuar a viver.

Uma das razões para que um mesmo evento gere consequências diferentes a determinadas pessoas relaciona-se ao conceito de resiliência (Benghozi, 2005; Pinheiro, 2004). Para Grotberb (1995) apud Mota, Benevides-Pereira, Gomes \& Araújo (2006, p. 58)

A resiliência pode ser definida como uma capacidade universal que possibilita a pessoa, grupo ou comunidade prevenir, minimizar ou superar os efeitos nocivos das adversidades, inclusive saindo dessas situações fortalecida ou até mesmo transformada, porém não ilesa.

O termo resiliência originou-se no âmbito da física e da engenharia, sendo conhecido há pouco tempo na área de Ciências Sociais e Humanas. Um material é denominado resiliente quando a energia de deformação máxima que ele é capaz de armazenar não gera nele deformações permanentes. Com esse conceito, é possível se fazer uma analogia ao termo utilizado pela física e pela psicologia: a relação tensão/ pressão com deformação não-permanente do material corresponderia à situação que ocorre entre uma situação de risco / estresse / experiências adversas/ respostas finais de adaptação. Infelizmente, a definição de resiliência em psicologia não é tão clara e precisa como na física, pois múltiplos fatores devem ser considerados no estudo dos fenômenos humanos (Barreira \& Nakamura, 2006; Yunes \& Szymanski, 2001).

Existem três tipos de resiliência de acordo com Garcia (2001), a emocional, a acadêmica e a social. A resiliência emocional relaciona as experiências positivas que levam a sentimentos de autoestima, autoeficácia e autonomia, que capacitam a pessoa a lidar com mudanças e adaptações, obtendo um repertório de abordagens para a solução de problemas. A resiliência acadêmica engloba a escola como um lugar onde habilidades para resolver problemas podem ser adquiridas com a ajuda dos agentes educacionais. E a resiliência social envolve fatores relacionados ao sentimento de pertencimento, supervisão de pais e amigos, relacionamentos íntimos, ou seja, modelos sociais que estimulem a aprendizagem de resolução de problemas.

Um dos primeiros autores a discutirem sobre o conceito de resiliência foi Frederic Flach, que em 1966 afirmou que para uma pessoa ser resiliente, dependerá de sua habilidade de reconhecer a dor pela qual está passando, perceber qual o sentindo que ela tem e tolerá-la durante um tempo até que seja capaz de resolver esse conflito de forma construtiva. $\mathrm{O}$ autor complementa que o termo não se relaciona somente com aspectos psicológicos, mas também aos aspectos físicos e fisiológicos (Flach, 1991).

Já no Brasil, os estudos sobre a resiliência são recentes. Um levantamento das publicações sobre o tema elaborado por Souza \& Cerveny (2006) mostra que os primeiros trabalhos sobre resiliência no país surgiram entre 1996 e 1998. A temática mais focada na época eram crianças expostas a situações de risco, fatores de proteção e vulnerabilidade psicossocial e perfil do executivo.

Atualmente os estudos sobre resiliência englobam várias áreas, como a espiritualidade (JaramilloVélez, Ospina-Muñoz, Cabarcas-Iglesias \& 
Humphereys, 2005), transtorno do déficit de atenção/ hiperatividade (Regalla, Guilherme \& Serra-Pinheiro, 2007), trabalhadores de chão de fábrica de indústrias montadoras de veículos e autopeças (Cimbalista, 2006) e professores (Howard \& Johnson, 2004).

\section{Fatores relacionados à resiliência}

É importante salientar que a resiliência não pode ser considerada um escudo protetor, que fará com que nenhum problema atinja essa pessoa, a tornando rígida e resistente a todas as adversidades. Não existe uma pessoa que É resiliente, mas sim a que ESTÁ resiliente. Esse é um processo dinâmico, $\mathrm{e}$ as influências do ambiente e do indivíduo relacionam-se de maneira recíproca, fazendo com que o indivíduo identifique qual a melhor atitude a ser tomada em determinado contexto (Pinheiro, 2004; Assis, Pesce \& Avanci, 2006).

Mota et al. (2006) citam Rutter (1985), que afirma que a resiliência envolve fatores de proteção e fatores de riso. Os fatores de proteção são as influências que modificam ou melhoram a resposta de uma pessoa a algum perigo que predispõe a um resultado não adaptativo, e que parecem mudar ou reverter circunstâncias potencialmente negativas. Relações parentais satisfatórias, disponibilidade de fontes de apoio social, autoimagem positiva, crença ou religião, etc, são considerados fatores de proteção.

Carvalho, Morais, Koller \& Peccinini (2007) afirmam que Eckenrode e Gore (1996) dividem os fatores de proteção em três classes: atributos pessoais, laços afetivos do sistema familiar, e contextos sociais que dêem suporte durante a adversidade, como a escola e o local de trabalho.

Os fatores de risco são variáveis que aumentam a probabilidade de desadaptação do sujeito diante de uma situação de perigo (Sapienza \& Pedromônico, 2005).

Vários autores enfatizam que os fatores de risco não são uma sentença (por exemplo, que todas as pessoas em situação de pobreza não serão resilientes), e que os fatores de proteção reduzem o impacto dos fatores de risco, reduzindo as reações negativas em exposição à situação de risco, estabelecendo a autoestima e criando oportunidades para reverter os efeitos do estresse. (Angst, 2008; Pesce, Assis, Santos \& Oliveira, 2004; Sapienza \& Pedromônico, 2005; Yunes, Garcia \& Albuquerque, 2007).
Outro fator associado à resiliência é a autoeficácia. Esse é um termo utilizado por Bandura, sendo a crença nas habilidades individuais de organizar e exercitar os recursos para administrar situações visando o futuro.

A resiliência e a autoeficácia percebida atuam como forma do sujeito obter uma melhor qualidade de vida na superação da adversidade, envolvendo o contexto, a cultura e a responsabilidade coletiva, sendo capaz de responderde diferentes formas ante um fracasso [...] (Barreira \& Nakamura, 2006, p. 78).

O conceito de coping também está diretamente ligado à resiliência, sendo que este caracteriza-se como o conjunto de estratégias utilizadas para a adaptação de circunstância adversas pelas pessoas. Lazarus e Folkman (1980) apud Antoniazzi, Dell'Aglio e Bandeira (1998) afirmam que o termo pode ser entendido de forma processual entre o indivíduo e o ambiente, tendo a função de administração de situações estressoras, sendo que o indivíduo deve avaliar e interpretar o fenômeno que é por ele percebido. Pessoas que se utilizam de estratégias de coping podem ser consideradas resilientes.

\section{Características relacionadas à resiliência}

Pessoas resilientes apresentam características básicas como: autoestima positiva, habilidades de dar e receber em relações humanas, disciplina, responsabilidade, receptividade, interesse, tolerância ao sofrimento e muitas outras (Barreira \& Nakamura, 2006).

Diversos estudos sobre o assunto apresentam situações que são consideradas fatores de risco, e como elas podem deixar de sê-lo através de estratégias adquiridas pelos indivíduos, geralmente composto por crianças e adolescentes (Bianchini \& Dell'Aglio, 2006; Castro \& MorenoJiménez, 2007; Paludo \& Koller, 2005; Peres, Mercante \& Nasello, 2005; Poletto, Wagner \& Koller, 2004; Sapienza \& Pedromônico, 2005; Schenker \& Minayo, 2005).

Grotberg (2005) identifica fatores resilientes e os divide em quatro categorias: "eu tenho", e "eu posso", "eu sou" e "eu estou". 


\begin{tabular}{|c|c|}
\hline Eu tenho: & Eu posso: \\
\hline $\begin{array}{l}\text { Pessoas do entorno em quem confio e que me } \\
\text { querem incondicionalmente; }\end{array}$ & Falar sobre coisas que me assustam ou inquietam; \\
\hline $\begin{array}{l}\text { Pessoas que me põem limites para que eu aprenda a } \\
\text { evitar os perigos ou problemas; }\end{array}$ & Procurar a maneira de resolver os problemas; \\
\hline $\begin{array}{l}\text { Pessoas que me mostram, por meio de sua conduta, } \\
\text { correta de proceder; }\end{array}$ & $\begin{array}{l}\text { Controlar-me quando tenho vontade de fazer algo a maneira } \\
\text { errado ou perigoso; }\end{array}$ \\
\hline $\begin{array}{l}\text { Pessoas que querem que eu aprenda a me } \\
\text { desenvolver sozinho; }\end{array}$ & Procurar o momento certo para falar com alguém; \\
\hline $\begin{array}{l}\text { Pessoas que me ajudam quando estou doente, ou } \\
\text { em perigo, ou quando necessito aprender. }\end{array}$ & Encontrar alguém que me ajude quando necessito. \\
\hline Eu sou: & Eu estou: \\
\hline Uma pessoa pela qual os outros sentem apreço e carinho; & Disposto a me responsabilizar por meus atos; \\
\hline $\begin{array}{l}\text { Feliz quando faço algo bom para os outros e lhes } \\
\text { demonstro meu afeto; }\end{array}$ & Certo de que tudo sairá bem \\
\hline Respeitoso comigo mesmo e com o próximo. & \\
\hline
\end{tabular}

FIGURA 1 - Fatores encontrados em pessoas resilientes Fonte: Retirado de Grotberg (2005, p. 17).

Laranjeira (2007) alerta que a maior parte dos estudos sobre resiliência são elaborados com crianças e adolescentes, havendo uma escassez de estudos com idosos. Esses poucos estudos levantam a questão se a resiliência é adquirida na infância ou se apenas algumas pessoas são resilientes e os que simplesmente não o são e nunca o serão. Com o envelhecimento surgem situações de risco, como a morte de pessoas próximas, doenças e aposentadoria, e que, diante delas, os idosos respondem de formas variadas, sendo resiliente ou não. O autor afirma que estudos transgeracionais deveriam ser efetuados para que se compreenda a perenidade ou não da resiliência.

\section{Resiliência, saúde e doença}

Um estudo elaborado por Mota et al. (2006) com portadores da Doença de Chagas sintomáticos e assintomáticos demonstrou que quem possuía sintomas da doença apresentava mais sintomas, tanto físicos como psicológicos de estresse, e menor capacidade de resiliência. Essa pesquisa aposta que a baixa resiliência predispõe o indivíduo ao desenvolvimento de sintomas de estresse, levando consequentemente à predisposição a sintomas da Doença de Chagas, o que gera assim uma maior morbidade.

Bianchini e Dell'Aglio (2006) afirmam que o sujeito passa por diversas situações na vida em que se deve apresentar a resiliência, mas a principal delas é a situação de doença. No contexto de enfermidade, a resiliência seria a capacidade de um indivíduo lidar com a doença aceitando as limitações que lhe são impostas diante de sua nova condição, colaborando com aderência ao tratamento e readaptando-se de forma positiva. Baseando-se nessa perspectiva, é preciso entender o paciente como único, pois sua história de vida irá determinar como irá defrontar-se com a doença. Dessa forma, nenhuma doença é a mesma para diferentes pessoas, sendo capaz de provocar reações diversas e singulares a cada um. 


\section{A medição da resiliência}

Apesar de existir um consenso sobre o que seja a resiliência e quais são as suas principais características, é de grande dificuldade quantificar aspectos relacionados a fatores de risco e proteção e quais as estratégias utilizadas por cada indivíduo para a resolução de problemas que seja capaz de denominá-los resilientes ou não. Por esse motivo, a construção de instrumentos de medição da resiliência é visto tanto com reserva quanto como necessidade para a facilitação e para a detecção de possíveis intervenções. Yunes e Szymanski (2001) advertem que não é possível se fazer inferências do tipo causa e efeito quando se trata de riscos psicológicos, sendo necessário identificar o ambiente como um todo, e não apenas o indivíduo. Já Pesce, Assis, Avanci, Santos, Malaquias e Carvalhaes (2005) afirmam que o aumento do interesse pelo conceito de resiliência ocorre atualmente como um reflexo da necessidade de projetos de intervenção e promoção da saúde mental, e por esse motivo torna-se necessário a criação de instrumentos que avaliem esse constructo.

Muñoz (2007) realizou uma revisão bibliográfica via internet de todos os estudos que abordavam o tema medição da resiliência. Foram encontrados ao todo 30 textos e foram categorizados em três áreas. A primeira área englobava as provas projetivas, que consistiam em mostrar histórias excessivamente problemáticas e demandantes a um grupo de adultos e pedir para que completassem o final da história. A segunda área referia-se às provas psicométricas, que em sua maioria eram autoinformes tipo likert e submetidas à análise fatorial. E a última área engloba as provas de imaginologia, que se baseiam em medições de potenciais eletroencefalográficos, provas neuroendocrinológicas, de sistema imune e exames genéticos, pois enfocam sua área de estudos no temperamento do indivíduo resiliente.

No Brasil, há apenas uma escala de resiliência validada e outra em fase de validação. Pesce et al. (2005) validaram para a população brasileira a Escala de Resiliência de Wagnild e Young, que é um inventário do tipo likert que varia de 1 (discordo totalmente) a 7 (concordo totalmente) e mede os níveis de adaptação psicossocial positiva.

Mota et. al. (2006) utilizaram-se do Inventário de Resiliência proposto por BenevidesPereira (2004) que está em fase de validação, sendo composto por 40 afirmações divididas em cinco fatores: desesperança e dificuldades emocionais, assertividade, tenacidade e inovação, empatia e sensibilidade emocional.

A utilização de testes para a medição da resiliência pode trazer muitas vantagens para pesquisadores da área, pois, se bem desenvolvidos, eles poderão auxiliar na detecção precoce de riscos existentes e poderá oferecer informações úteis para estabelecer diagnósticos e planos de intervenção.

\section{Contribuição do conceito de resiliência para a psicologia}

Ao passar por uma situação traumática, muitas pessoas tendem a procurar ajuda profissional para orientá-lo nesse contexto adverso. O psicólogo é um desses profissionais que possui formação para disponibilizar-se a tal ajuda.

Peres et al. (2005) afirmam que a finalidade da psicoterapia para um sujeito que foi vítima de um trauma psicológico é lhe atribuir novos significados emocionais à situação traumática que não ocorre mais no presente. É importante o psicólogo compreender que um evento é traumático para uma pessoa, e que para outra pode não ser. O estímulo estressor vai ser visto de diferentes formas por diversas pessoas, e o que o caracteriza como estressor é o processamento perceptual do indivíduo, ou seja, como ele interpretou os fatos que lhe ocorreram naquele momento. "Aprender e crescer a partir das experiências positivas e negativas de vida e desenvolver a capacidade de lidar com adversidades severas são aspectos cruciais a serem trabalhados em psicoterapia" (Peres et al., 2005, p. 136).

É de grande importância salientar que não se deve apenas focar em um indivíduo e denominá-lo resiliente ou não. Suas características resilientes ou não resilientes estão também relacionadas ao ambiente que o cerca, e rotular cada pessoa pode causar maiores preconceitos e limitações em intervenção a serem realizadas.

O uso indiscriminado do termo pode acarretar preconceitos, prejudicando tanto a pessoa que foi rotulada, quanto o trabalho do psicólogo, pois o profissional pode sentir-se incapacitado de realizar algo para auxiliar e reverter a situação na qual se encontra certo indivíduo ou população.

A resiliência pode ser compreendida nos diversos campos da Psicologia, sendo que independente do contexto no qual o psicólogo 
trabalha, há a possibilidade de antes de ver os aspectos de risco e que devem ser melhorados, ver quais são as qualidades e aspectos a serem enfatizados para a valorização do que já existe e que pode auxiliar na mudança de situações atuais.

Atualmente estão surgindo estudos que vêm contribuindo para a mudança de foco dos profissionais psicólogos, sendo esse o conceito de psicologia positiva. A formação em Psicologia tende a capacitar os profissionais a lidar de forma satisfatória com a doença, os desajustes e os conflitos, porém, o psicólogo não apresenta subsídios necessários para focar seu trabalho nos aspectos sadios e de sucesso de determinado grupo.

Essa é a proposta da psicologia positiva, que visa focar-se no que está dando certo em determinado grupo ou indivíduo, e não apenas no que deu errado e é disfuncional (Aznar-Farias \& Oliveira-Monteiro, 2006; Carvalho, Calvo, Campos \& Castillo, 2006; Howard \& Johnson, 2004; Ravazzola, 2005). Yunes (2003) afirma que a psicologia positiva objetiva romper com o viés reducionista e negativo hoje existente, focalizando as potencialidades e qualidades humanas, sendo a resiliência uma forma de considerar-se os fenômenos indicativos de uma vida saudável e adaptativa ao longo do desenvolvimento.

A mudança do paradigma atual existente, que enfatiza apenas os aspectos patológicos, deve ser mudada para que se entenda um indivíduo como alguém que está muito além de sua queixa e de seus problemas, mas também de seus aspectos saudáveis e adaptativos.

\section{CONSIDERAÇÕES FINAIS}

Os estudos sobre a resiliência são de grande contribuição para o trabalho do psicólogo. Com o conhecimento da resiliência pode-se começar a ver um indivíduo como capaz de procurar recursos para superar as adversidades, não sendo apenas mais um observador passivo e "doente", e sim como alguém que pode buscar recursos em si mesmo e no ambiente que o rodeia para a resolução de conflitos.

O tema não está esgotado, pois há questões que devem ser mais estudadas para que seja possível compreender como se deve intervir para a promoção da resiliência desde a infância para que os resultados se reflitam na vida adulta. Sapienza e Pedromônico (2005, p. 213) enfatizam que "A identificação de como e em que fase do desenvolvimento atuam os mecanismos protetores é fundamental para a organização de intervenções efetivas para a redução de problemas de comportamento."

É de extrema importância compreender que a resiliência não é adquirida, e sim aprendida de diversas formas, sendo uma delas a realização de programas voltados a diferentes populações. Desta forma, mostra-se essencial a participação tanto de instituições como de profissionais para a promoção da resiliência.

A formação recebida pelos profissionais também deve ser repensada, pois é de grande urgência que se enfatizem outros aspectos além dos relacionados a patologias e problemas decorrentes do mesmo. É preciso ter uma visão global de cada pessoa, observando suas estratégias e relacionamentos interpessoais.

A metáfora que melhor explica o processo de resiliência é o da ostra: para protegerse do grão de areia que a fere, envolve de nácar o intruso, arredondando as asperezas, dando origem a uma pérola, uma bela joia preciosa (LARANJEIRA, 2007). Assim ocorre com o indivíduo: quando se defronta com uma situação adversa, ao tomar atitudes resilientes, sai da situação mais fortalecido e belo do que era antes.

\section{AGRADECIMENTOS}

Agradeço a constante supervisão e apoio do professor Cloves Amorim.

\section{REFERÊNCIAS}

Angst, R. (2008). Resiliência: Qual a contribuição para a Psicologia? Trabalho apresentado no XVII Encontro Brasileiro de Psicoterapia e Medicina Comportamental, Campinas: ABPMC.

Assis, S. G., Pesce, R. P., \& Avanci, J. Q. (2006). Resiliência: enfatizando a proteção dos adolescentes. Porto Alegre: Artmed.

Antoniazzi, A. S., Dell'Aglio, D. D., \& Bandeira, D. R. (1998) O conceito de coping: uma revisão teórica. Estudos em Psicologia, 3(2), 273-294. 
Aznar-Farias, M., \& Oliveira-Monteiro, N. R. (2006). Reflexões sobre pró-sociabilidade, resiliência e psicologia positiva. Revista Brasileira de Terapia Cognitiva, 2(2), 39-46.

Barreira, D. D., \& Nakamura, A. P. (2006). Resiliência e a auto-eficácia percebida: Articulação entre conceitos. Aletheia, 23, 75-80.

Benghozi, P. (2005). Resiliência familiar e conjugal numa perspectiva psicanalítica dos laços. Psicologia Clínica, 17(2), 101-109.

Bianchini, D. C. S., \& Dell'Aglio, D. D. (2006). Processos de resiliência no contexto de hospitalização: Um estudo de caso. Paidéia, 16(35), 427-436.

Carvalho, V. A. M. L., Calvo, B. F., Martín, L. H., Campos, F. R., \& Castillo, I. C. (2006). Resiliencia y el modelo burnout-engagement en cuidadores formales de ancianos. Psicothema, 18(4), 791-798.

Carvalho, F. T., Morais, N. A., Koller, S. H., \& Piccinini, C. A. (2007). Fatores de proteção relacionados à promoção de resiliência em pessoas que vivem com HIV/AIDS. Caderno de Saúde Pública, 23(9), 2023-2033.

Castro, E. K., \& Moreno-Jiménez, B. (2007). Resiliencia en niños enfermos crónicos: Aspectos teóricos. Psicología em Estudo, 12(1), 81-86.

Cimbalista, S. (2006). Reflexões sobre o trabalho e a subjetidade de trabalhadores resilientes sob o sistema de produção flexível. Revista da FAE, 9(2), 13-28.

Flach, F. F. (1991). Resiliência: A arte de ser flexível. São Paulo: Saraiva.

Garcia, I. (2001). Vulnerabilidade e resiliência. Adolescencia Latinoamericana, 2, 128-130.

Grotberg, E. H. (2005). Introdução: novas tendências em resiliência. In: A. Melillo \& E. N. S. Ojeda (Org.). Resiliência: Descobrindo as próprias fortalezas (pp. 15-22). Porto Alegre: Artmed.

Howard, S., \& Johnson, B. (2004). Resilient teachers: Resisting stress and burnout. Social Psychology of Education, 7, 399-420.

Jaramillo-Vélez, D. E., Ospina-Muñoz, D. E., Cabarcas-Iglesias, G., \& Humphereys, J. (2005). Resiliencia, espiritualidade, aflicción y tácticas de resolución de conflictos en mujeres maltratadas. Revista de Saúde Pública, 7(3), 281-292.
Laranjeira, C. A. S. J. (2007). Do vulnerável ser ao resiliente envelhecer: Revisão de literatura. Psicologia: Teoria e Pesquisa, 23(3), 327-332.

Mota, D. C. G. A., Benevides-Pereira, A. M. T., Gomes, M. L., \& Araújo, S. M. (2006). Estresse e resiliência em doença de chagas. Aletheia, 24, 57-68.

Muñoz, D. E. O. (2007). La medición de la resiliencia. Investigación y Educación en Enfermería, 25(1), 58-65.

Paludo, S. S., \& Koller, S. H. (2005). Resiliência na rua: Um estudo de caso. Psicologia: Teoria e Pesquisa, 21(2), 187-195.

Peres, J. F. P., Mercante, J. P. P., \& Nasello, A. G. (2005). Promovendo resiliência em vítimas de trauma psicológico. Revista de Psiquiatria RS, 27(2), 131-138.

Pesce, R. P, Assis, S. G., Santos, N., \& Oliveira, R. V. C. (2004). Risco e proteção: Um equilíbrio promotor de resiliência. Psicologia: Teoria e Pesquisa, 20(2), 135-143.

Pesce, R. P., Assis, S. G., Avanci, J. Q., Santos, N. C., Malaquias, J. V., \& Carvalhaes, R. (2005). Adaptação transcultural, confiabilidade e validade da escala de resiliência. Caderno de Saúde Pública, 21(2), 436-448.

Pinheiro, D. P. N. (2004). A resiliência em discussão. Psicologia em Estudo, 9(1), 67-75.

Poletto, M., Wagner, T. M. C., \& Koller, S. H. (2004). Resiliência e desenvolvimento infantil de crianças que cuidam de crianças: Uma visão de perspectiva. Psicologia: Teoria e Pesquisa, 20(3), 241-250.

Ravazzola, M. C. (2005). Resiliências familiares. In A. Melillo, E. N. S. Ojeda (Org.). Resiliência: Descobrindo as próprias fortalezas (pp. 73-85). Porto Alegre: Artmed.

Regalla, M. A., Guilherme, P. R., \& Serra-Pinheiro, M. A. (2007). Resiliência e transtorno do déficit de atenção/hiperatividade. Jornal Brasileiro de Psiquiatria, 56(supl. 1), 45-49.

Sapienza, G., \& Pedromônico, M. R. M. (2005). Risco, proteção e resiliência no desenvolvimento da criança e do adolescente. Psicologia em Estudo, 10(2), 209-216.

Schenker, M., \& Minayo, M. C. S. (2005). Risk and protective factors and drug use among adolescence. Ciência Saúde Coletiva, 10(3), 707-717. 
Souza, M. T. S., \& Cerveny, C. M. O. (2006). Resiliência psicológica: Revisão de literatura e análise de produção científica. Revista Interamericana de Psicologia. 40(1), 119-126.

Yunes, M. A. M., \& Szymanski, H. (2001). Resiliência: Noção, conceitos afins e considerações críticas. In J. Tavares (Org.). Resiliência e educação (pp.13-42). São Paulo: Cortez.

Yunes, M. A. M. (2003). Psicologia positiva e resiliência: O foco no indivíduo e na família. Psicologia em Estudo, 8 (N. especial), 75-84.

Yunes, M. A. M., Garcia, N. M., \& Albuquerque, B. M. (2007). Monoparentalidade, pobreza e resiliência: entre as crenças dos profissionais e as possibilidades da convivência familiar. Psicologia: Reflexão e Crítica, 20(3), 444-453.

Recebido: 05/12/2008 Received: 12/05/2008

Aprovado: 10/01/2009 Approved: 01/10/2009

Revisado: 00/00/0000

Reviewed: 00/00/0000 